\section{Combining CoMP with semi-smart antennas to improve performance}

\section{Yang, Y. Wang, T. Zhang, L. Cuthbert and L. Xiao}

A new approach for improving the throughput and reducing the transmit power of OFDMA networks is presented. This combines Co-ordinated Multi-Point (CoMP) with variable cell radiation patterns from semi-smart antennas to optimise the network configuration leading to greater throughput for lower transmission power compared with CoMP on its own.

Introduction: It is well known that cell-edge users in OFDMA cellular networks suffer poorer SINR because of (i) the greater path loss to the base station (BS) and (ii) increased interference from neighbouring cells. Co-ordinated Multi-Point (CoMP) [1] has been proposed as a technique to overcome that problem. CoMP is a distributed MIMO approach that changes the interference signal from neighbouring base stations into a useful signal; it does this by ensuring the signals from base stations are transmitted in a cooperative way. This does require the BSs to be synchronised and to cooperate tightly in order to create MIMO channel coding. The remote synchronising and cooperating make CoMP much more complicated than MIMO and having the same information transmitted from different BSs takes up system resource. The most likely case is when two BSs cooperate.

The users that are close to a BS or that are receiving a high SINR do not need CoMP; only users that are located at the cell edge and suffer low SINR require it. The choice of which users are treated as CoMP users depends on the channel state: SINR, distance to the BS, or the useful signal received power.

Cooperative semi-smart antennas, originally proposed in 2001 for WCDMA systems [2], have been proposed as another technique for improving the SINR for cell-edge users in OFDMA systems [3], by changing the radiation patterns to minimise interference. The semismart antenna system gives a broadly-shaped radiation pattern that can cover a large area and as there is no expensive DSP needed to track individual users, the cost of a semi-smart antenna is much lower than a fully adaptive antenna system. By changing these broad patterns in a co-ordinated way it is possible to optimise the network to minimise interference and maximise capacity, without there being any 'holes' in coverage, as reported in [3].

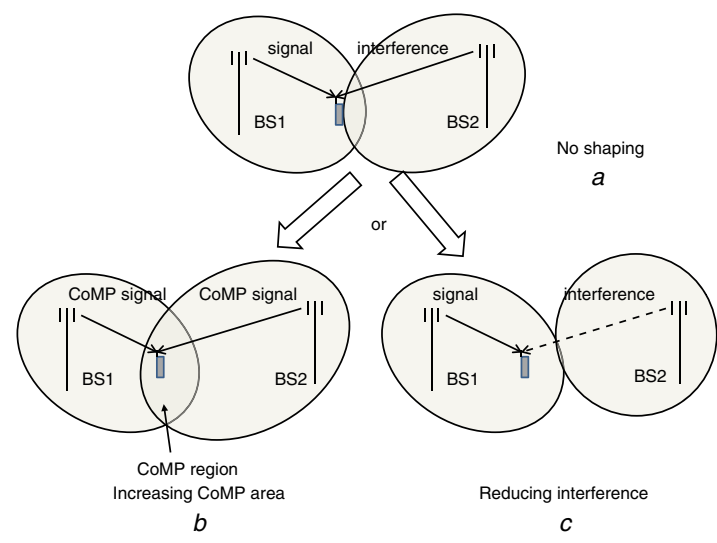

Fig. 1 Principles of combining semi-smart antennas with CoMP

Combining CoMP with smart antennas: In this Letter we go beyond the current work by adding CoMP to the semi-smart antennas to give an extra degree of freedom to the interference mitigation. As explained later, the results are significant.

While there has been some work on beamforming with MIMO (e.g. [4]) there has been no previous work on combining co-operative semismart antennas with CoMP.

Fig. 1 shows the principle behind the combination with two cooperating BSs. In Fig. 1 $a$ a cell-edge user is located just outside the CoMP region, but enlarging the radiation pattern of BS2 could extend the CoMP region and bring the user into that, as shown in Fig. $1 b$. This brings an extra degree of freedom to the solution, but also extra complexity: while extending the CoMP region is one solution for that particular UE, so would be shrinking the pattern of BS2 to reduce the interference, as shown in Fig. 1c. Of course, changing the radiation pattern will effectively change the channel state between the BSs affected and all the UEs in that cell. The combination is complex but effective.

The approach used here to demonstrate the benefit that can be obtained is to optimise the radiation patterns:

(i) subject to the constraint that the whole area must be covered and there should be no 'holes'; and

(ii) with an objective function that maximises the overall network capacity and minimises RF transmitting power.

By applying CoMP with a standard algorithm (using the SINR seen by the user) to each generated set of antenna patterns and then optimising the antenna patterns, we avoid making the decision whether to follow the extended CoMP of Fig. $1 b$ or to reduce the interference of Fig. $1 c$ for any particular user: this is handled within the optimisation.

Simulation: Genetic algorithms (GAs) [5] are well-known effective search algorithms to find a near-optimal solution for many fields where little knowledge is known or there are many conflicting constraints or objectives to affect best solutions. In this case, using GAs allows us to learn the best radiation patterns for a system using CoMP, at the same time satisfying the constraint that the whole region is covered.

The outline of the approach is as follows:

(i) Choose a starting set of radiation patterns.

(ii) For each pattern determine the performance of the network using CoMP.

(iii) Use the GA to select and modify the radiation patterns according to the performance function and subject to the coverage constraint.

(iv) Repeat this loop until the results are stable, when the radiation patterns are the best that the GA can find for that particular distribution of users.

The advantage of this approach is that it separates the calculation of the radiation patterns from the detail of allocating resources to users and deciding whether each one is a CoMP or normal user; the disadvantage is that it is a lengthy process, but it does illustrate the advantage that can be obtained by combining the two techniques and sets a benchmark for faster algorithms.

Results: The results for a representative user distribution containing hotspots mixed with uniformly distributed users are shown in Fig. 2. Clearly the use of CoMP and of semi-smart antennas each leads to improved performance, but the improvement with the combination of the two techniques is clearly superior; in this case an improvement in throughput of $25 \%$ over the case with fixed antennas, or a reduction in power by $50 \%$ to achieve the same throughput. Obviously, the exact gain will depend on the user distribution, but as discussed below the method is not sensitive to changes in distribution.

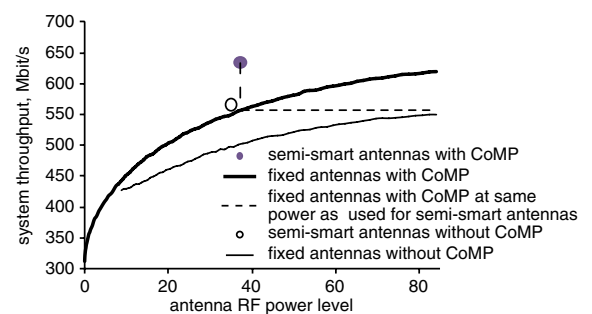

Fig. 2 Results showing benefit of combination

In a mobile network users will move; with semi-smart antennas there is no concept of following an individual user (unlike the smart antennas in TD-SCDM for example). Moreover, rapid changes in the effective shape of a cell should be avoided as this will increase the frequency of handovers. Fig. 3 shows the change in throughput as users move within the optimal antenna patterns from Fig. 2. It is assumed that $30 \%$ of the users move at a speed of $3 \mathrm{~km} / \mathrm{h}$, the value commonly used for pedestrians, and the cell radius is $500 \mathrm{~m}$. This is an urban scenario and it is assumed that moving vehicles are handled by an overlay macro-cell. The change in throughput (recalculating CoMP as the 
users move) is shown in Fig. 3 for a time period of up to $100 \mathrm{~s}$. It is clear that the sensitivity to movement is low so that: (i) there is no need for rapid changes in antenna patterns; (ii) the throughput is greater than that which would be obtained from the same power but no smart antennas (the dashed line in Fig. 3); and (iii) a fast algorithm is not required.

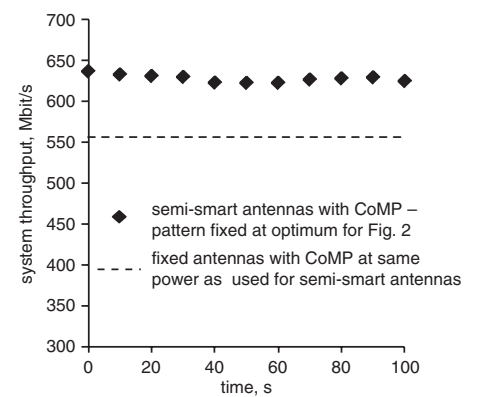

Fig. 3 Sensitivity to movement of $30 \%$ of UEs

Conclusion: We have shown that combining semi-smart antennas with CoMP can lead to a significant improvement in performance for OFDMA cellular systems compared with that which can be achieved with either technique on its own. This improvement in performance can be seen in terms of throughput and reduced transmission power. Initial studies on sensitivity to movement of UEs shows that the approach is robust, not requiring rapid changes in antenna radiation patterns yet still achieving better throughput compared with just using CoMP on its own.
(C) The Institution of Engineering and Technology 2011

16 March 2011

doi: $10.1049 / \mathrm{el} .2011 .0736$

One or more of the Figures in this Letter are available in colour online.

$\mathrm{X}$. Yang and Y. Wang (MPI-QMUL Information Systems Research Centre, Macao Polytechnic Institute, Macao SAR, China)

E-mail: xuy@mpi-qmul.org

T. Zhang (School of Information and Communication Engineering, Beijing University of Posts and Telecommunications, Beijing, People's Republic of China)

L. Cuthbert (School of Electronic Engineering and Computer Science, Queen Mary University of London, London E1 4NS, United Kingdom)

L. Xiao (Nanchang University, Jiangxi, People's Republic of China)

\section{References}

1 Karakayali, M.K., Foschini, G.J., and Valenzuela, R.A.: 'Network coordination for spectrally efficient communications in cellular systems', IEEE Wirel. Commun., 2006, 13, pp. 56-61

2 Bigham, J., Parini, C., and Cuthbert, L.: inventors; Queen Mary and Westfield College, assignee. Intelligent control of radio resources in a wireless network. United States patent US 7,570,956, 4 Aug. 2009

3 Yang, X., Wang, Y., Zhang, D., and Cuthbert, L.: 'Resource allocation in LTE OFDMA systems using genetic algorithm and semi-smart antennas'. Proc. IEEE Wireless Communications and Networking Conf., (WCNC), 2010, Sydney, Australia, April 2010, pp. 1-6

4 Mundarath, J., and Kotecha, J.: 'Optimal receive array beamforming for non-collaborative MIMO space division multiple access', IEEE Trans. Commun., 2010, 58, (1), pp. 218-227

5 Mitchell, M.: 'An introduction to genetic algorithms' (MIT Press, Massachusetts, 1998) 